\title{
Avaliação do Consumo e Ganho de Peso de Novilhos em Pastejo Rotacionado de Capim-Elefante (Pennisetum purpureum, Schum.) cv. Napier, na Estação Chuvosa 1
}

\author{
Eleonora D’Avila Erbesdobler ${ }^{2}$, Carlos Augusto de Alencar Fontes ${ }^{3}$, Domingos Sávio Queiroz ${ }^{4}$, \\ Hernan Maldonado Vasquez ${ }^{5}$, Manoel Messias Pereira da Silva ${ }^{6}$, Ana Cristina Ladeira de \\ Souza Gesualdi ${ }^{6}$, José Antônio de Freitas ${ }^{7}$
}

\begin{abstract}
RESUMO - A pesquisa foi conduzida na Fazenda Experimental da EPAMIG-Leopoldina, MG, onde foram avaliados o consumo de capim-elefante, a efetividade da limitação do tempo de pastejo a quatro horas diárias, para manter o consumo próximo à mantença e o ganho de peso de 36 novilhos 3/4 Gir-Holandês, em cinco períodos sucessivos de 36 dias, na estação chuvosa. Os tratamentos foram: livre acesso ao pasto (pastejo irrestrito) e pastejo restrito. A disponibilidade de matéria verde seca do capim-elefante nos cinco períodos variou de 4,0 a 7,8 kg/100 kg PV. Os animais com livre acesso ao pasto consumiram o equivalente a 1,61 vezes o dos animais de pastejo restrito e tiveram ganho de peso de corpo vazio médio diário de 516,4 g, com um ganho estimado de 432,5 kg por hectare, estando dentro da faixa de ganho de peso verificada para capim-elefante no Brasil. O consumo de energia metabolizável por unidade de tamanho metabólico manteve-se estável nos três primeiros períodos, elevando-se nos dois últimos períodos, em que ocorreram os maiores ganhos de peso dos animais. Quatro horas diárias de pastejo garantiram consumo próximo à mantença.
\end{abstract}

Palavras-chave: disponibilidade, pastejo irrestrito, pastejo restrito

\section{Evaluation of Intake and Weight Gain of Steers in Rotacional Grazing of Elephantgrass (Pennisetum purpureum, Schum.) cv. Napier, during the Rainy Season}

\begin{abstract}
The research was conducted in the experimental farm of the EPAMIG-Leopoldina, MG, to evaluate intake of elephantgrass, the effectiveness of limiting grazing time to four daily hours for keeping intake close to maintenance level, and the weight gain of thirty-six steers during five successive periods of thirty-six days, during the rainy season. The treatments were: free access to pasture (unlimited grazing) and restricted grazing. The availability of green dry matter varied from 4.0 to $7.8 \mathrm{~kg} / 100 \mathrm{~kg} \mathrm{LW}$. The average daily weight gain of animals with free access to pasture was $516.5 \mathrm{~g}$ and their dry matter intake corresponded to 1.61 times that from the restricted grazing ones. The estimated gain per hectare was $423.5 \mathrm{~kg}$, which is within the range reported for elephantgrass in Brasil. Metabolizable energy intake, per unit metabolic size, remained stable in the first three periods and increased in the last two, during which the highest weight gains of the animals. Four daily hours grazing period ensured intake close to maintenance level.
\end{abstract}

Key Words: availability, unlimited grazing, restricted grazing

\section{Introdução}

O modelo extensivo de produção de bovinos a pasto prevalece no Brasil, o que está associado às características climáticas, extensão territorial e aos altos custos dos grãos, em relação ao preço da carne. É pequena a parcela de animais para abate que recebe suplementação protéica e energética nas fases de crescimento e engorda, sendo mais comum o uso apenas de suplementos minerais. A utilização de forrageiras com potencial elevado de crescimento e bom valor nutritivo possibilita maiores ganhos diários, por animal e por área, durante a estação chuvosa, elevando-se o ganho médio anual, mesmo quando ocorrem baixos ganhos na seca.

O capim-elefante é considerado a espécie com maior potencial de produção, encontrando-se na literatura referência de produção de até 80 toneladas de matéria seca por ha, por ano (Santos, 1995). Em uma revisão sobre o uso da citada forrageira para produ-

\footnotetext{
${ }^{1}$ Parte do trabalho de tese de mestrado do primeiro autor, financiado com recursos da Fundação Estadual do Norte Fluminense (FENORTE) e da Empresa de Pesquisa Agropecuária de Minas Gerais (EPAMIG).

2 Zootecnista, M.Sc., Laboratório de Ciências Ambientais (LCA), Centro de Biociências e Biotecnologias (CBB), Universidade Estadual do Norte Fluminense (UENF), Av. Alberto Lamego, 2000, Campos dos Goytacazes, RJ.CEP - 28015-620. E.mail: lola@uenf.br

3 Professor Titular do Laboratório de Zootecnia e Nutrição Animal (LZNA), Centro de Ciências e Tecnologias Agropecuárias (CCTA),

Universidade Estadual do Norte Fluminense. E.mail: cafontes@uenf.br

4 Pesquisador da EPAMIG/CTZM, Viçosa-MG. E.mail: dqueiroz@mail.ufv.br

5 Professor Associado do LZNA/CCTA/UENF. E.mail: hernan@uenf.br

6 Zootecnista, M.Sc., LZNA/CCTA/UENF. E.mail: manel@uenf.br

7 Professor - Universidade Federal do Paraná (UFPR).
} 
ção de carne, sob pastejo, Veiga (1994) afirma que o volume de informações disponíveis é ainda bastante restrito e Deresz \& Mozzer (1994), com base nos dados disponíveis, concluem que o capim-elefante pode proporcionar altos ganhos por área, principalmente devido à sua elevada capacidade de suporte e aos bons ganhos de peso proporcionados.

O fenômeno do ganho compensatório ocorre de forma significativa no início do período chuvoso, com possível elevação do ganho de peso. Esse fenômeno é explorado como parte integrante do processo de produção de bovinos em regime de pasto, em vários países. Nesse sentido, Nicol \& Kitessa (1995) afirmam que esse ganho é inerente ao sistema de produção de gado de corte na Nova Zelândia, sendo compatível com o comportamento sazonal de produção da pastagem.

O nível de consumo de forragem, no período de maior crescimento vegetativo, responde por $75 \%$ da variação entre forragens, quanto ao desempenho animal (Minson, 1990).

O trabalho foi conduzido com os objetivos de se determinar o consumo do capim-elefante e avaliar o ganho de peso de novilhos, ao longo do período de maior crescimento vegetativo da gramínea, em regime de pastejo, além de verificar a efetividade da restrição do número de horas de pastejo como forma de garantir o consumo alimentar próximo ao nível de mantença, de forma a subsidiar futuros estudos de determinação de exigências de energia de mantença de bovinos, em regime de pasto.

\section{Material e Métodos}

O trabalho foi conduzidona Fazenda Experimental da EPAMIG, emLeopoldina, MG, com duração de 175 dias. Foram utilizados 36 novilhos $3 / 4$ Gir-Holandês com idade inicial média de 20 meses e peso vivo(PV) médio de $332 \mathrm{~kg}$. Dezoito animais receberam um dos tratamentos: 1) pastejo irrestrito (PI), com livre acesso ao pasto e 2) pastejo restrito (PR), limitado a 4 horas por dia. Utilizaram-se nove piquetes de capim-elefante (Pennisetum purpureum, cv. Napier), já existentes, com área individual de 0,5 ha, em pastejo rotacionado com período de ocupação de 4 dias, descanso de 32 dias e taxa de lotação próxima a 5 animais por ha. Para fim de ocupação dos piquetes, os animais dos dois tratamentos constituíram grupo único, pastejando o mesmo piquete. Estimou-se que o consumo de um animal com livre acesso ao pasto equivaleria a 1,6 vezes o do animal de pastejo restrito, no cálculo da taxa de lotação. O consumo alimentar dos animais dos dois tratamentos foi estimado seis vezes durante o período experimental, de forma a se obter a relação real entre o consumo dos dois tratamentos. Animais semelhantes aos experimentais foram introduzidos, para substituírem animais abatidos ao longo da pesquisa. Foram utilizados quatro animais fistulados no esôfago pela técnica descrita por Bishop \& Froseth (1970), para obtenção de amostras de extrusa, em coletas mensais com bolsas coletoras feitas de lona impermeável, durante oito dias consecutivos, nos cinco períodos de pastejo de 36 dias.

A produção fecal foi estimada seis vezes durante o período experimental, fornecendo-se $5 \mathrm{~g}$ de óxido de cromo $\left(\mathrm{Cr}_{2} \mathrm{O}_{3}\right)$ aos animais, duas vezes ao dia, durante 16 dias. Amostras fecais foram obtidas por coleta retal, duas vezes ao dia, no momento do fornecimento de $\mathrm{Cr}_{2} \mathrm{O}_{3}$. As amostras individuais foram colocadas em sacos plásticos e, ao final do período de coleta, foram homogeneizadas, obtendo-se amostras compostas por animal, que foram armazenadas em congelador a $-15^{\circ} \mathrm{C}$ para posteriores análises laboratoriais.

A digestibilidade da forragem foi determinada pelo método in vitro (Tilley \& Terry, 1963), utilizando-se as amostras de extrusa anteriormente referidas. O consumo individual diário de energia digestível, por período, foi estimado baseando-se nos conteúdos de energia bruta da extrusa e das amostras compostas individuais de fezes, bem como dos valores diários individuais estimados de consumo de MS e de excreção fecal de MS. No cálculo de energia metabolizável(EM), adotou-se a relação $\mathrm{EM}=0,82 * \mathrm{ED}$ (NRC, 1996). A cada abate determinou-se o peso de corpo vazio (PCVZ) dos animais. A relação PCVZ/PV dos animais obtidos no início do experimento foi utilizada para estimar o PCVZ inicial dos animais experimentais, permitindo o cálculo dos ganhos de PCVZ e a estimativa do consumo de energia em relação ao PCVZ.

Tendo como um dos objetivos do experimento o conhecimento da eficiência da restrição alimentar como forma de garantir o consumo próximo ao nível de mantença e as variações das médias referentes ao consumo alimentar nos períodos experimentais, optou-se por desdobrar as interações tratamento $\mathrm{x}$ período sempre que fosse significativo ao nível de $10 \%$ de probabilidade. As variáveis consumo total, por $100 \mathrm{~kg}$ de peso vivo, por unidade de tamanho metabólico $\left(\mathrm{kg}^{0,75}\right)$ e o ganho de peso dos animais, ao longo da estação de pastejo, foram analisadas de acordo com o modelo:

R. Bras. Zootec., v.31, n.5, p.2123-2128, 2002 


$$
\mathrm{Y}_{\mathrm{ijkl}}=\mu+\mathrm{C}_{\mathrm{i}}+\mathrm{B}_{\mathrm{j}}+\mathrm{N}_{\mathrm{k}}+\mathrm{CB}_{\mathrm{ij}}+\mathrm{CN}_{\mathrm{ik}}+\mathrm{e}_{\mathrm{ijkl}}
$$

em que: $\mathrm{Y}_{\mathrm{ijkl}}=$ observação referente ao animal 1 , no período i, do bloco j, submetido ao nível de oferta k; $\mu=$ média geral; $\mathrm{C}_{\mathrm{i}}=$ efeito do ciclo (período) de pastejo $\mathrm{i}$, em que $\mathrm{i}=1,2,3,4$ e $5 ; \mathrm{B}_{\mathrm{j}}=$ efeito do bloco $\mathrm{j}$, sendo $\mathrm{j}=1,2,3 ; \mathrm{N}_{\mathrm{k}}=$ efeito de nível de oferta $\mathrm{k}$, sendo $\mathrm{k}=1$ irrestrito e 2 restrito; $\mathrm{CB}_{\mathrm{ij}}=$ efeito da interação entre o ciclo de pastejo i e o bloco j; $\mathrm{CN}_{\mathrm{ik}}=$ efeito da interação entre o ciclo de pastejo i e o nível de oferta $\mathrm{k} ; \mathrm{e}_{\mathrm{ijkl}}=$ erro aleatório, associado a cada observação, pressuposto normal e independentemente distribuído, com média zero e variância $\sigma^{2}$.

Para as análises estatísticas, utilizou-se o programa Least-Squares and Maximum Likelihood, versão PC-1, Harvey (1990). As médias foram comparadas pelo teste de Newman-Keuls, em nível de 5\% de probabilidade.

\section{Resultados e Discussão}

A pressão de pastejo mostrou-se dentro dos limites de 4,0 a 7,8 $\mathrm{kg}$ de matéria verde seca (MVS) por $100 \mathrm{~kg}$ de peso vivo (PV), situando-se, em três períodos, acima de $6 \mathrm{~kg}$ de MVS/100 kg de PV. Estudando a influência de diferentes pressões de pastejo sobre o consumo, Hillesheim (1987) concluiu que a pressão de pastejo, para capim-elefante, de $4 \mathrm{~kg} / 100 \mathrm{~kg}$ de PV de MS acumulada no período de pastejo, foi adequada, resultando em consumo de forragem semelhante às pressões de 8 e $12 \mathrm{~kg}$ de matéria seca (MS)/100 kg de PV. Porém, Stockdale \& King (1983) observaram que o consumo tendia a se estabilizar quando a oferta de forragem atingia três vezes o requerimento de consumo de MS, implicando uma pressão de pastejo de 6 a 9\% do PV para ocorrência do consumo máximo. A disponibilidade de MVS/animal/dia, para a planta inteira, variou de 13,8 no primeiro período de pastejo a $31,8 \mathrm{~kg}$ no quinto período, com período de descanso de 32 dias. A maior produção de MS disponível ocorreu no fim do período experimental, em que a temperatura ambiente elevada e o bom nível de água residual no solo proporcionaram elevado crescimento do capim.

O consumo médio total diário de MS dos animais em pastejo irrestrito, nos cinco períodos de pastejo, equivaleu a 1,61 vezes o dos animais de pastejo restrito, estando próxima à relação previamente estimada de 1,6 , observando-se no primeiro período o menorconsumo

Tabela 1 - Consumo médio total diário de matéria seca e matéria orgânica ( $\mathrm{kg} / \mathrm{dia}$ ) dos animais dos tratamentos PI (pastejo irrestrito) e PR (pastejo restrito) e relação entre as médias dos dois tratamentos, nos cinco períodos de pastejo

Table 1 - Average total daily intake of dry matter and organic matter ( $\mathrm{kg} /$ day) of the animals from the treatments UG (unrestricted grazing) and $R G$ (restricted grazing) and relation among the means for the two treatments, in five grazing periods

\begin{tabular}{|c|c|c|c|c|c|c|}
\hline \multirow{2}{*}{$\begin{array}{l}\text { Tratamentos } \\
\text { Treatments }\end{array}$} & \multicolumn{5}{|c|}{$\begin{array}{c}\text { Períodos } 1 \\
\text { Periods } 1\end{array}$} & \multirow{2}{*}{$\begin{array}{c}\text { Médias } \\
\text { Means }\end{array}$} \\
\hline & I & II & III & IV & $\mathrm{V}$ & \\
\hline \multicolumn{7}{|c|}{ Consumo de MS (kg) } \\
\hline $\mathrm{PI}(U G)$ & $7,48^{\mathrm{cA}}$ & $7,77^{\mathrm{bcA}}$ & $7,98^{\mathrm{bcA}}$ & $8,55^{\mathrm{abA}}$ & $9,12^{\mathrm{aA}}$ & 8,18 \\
\hline $\mathrm{PR}(R G)$ & $4,70^{\mathrm{bB}}$ & $4,98^{\mathrm{abB}}$ & $5,60^{\mathrm{aB}}$ & $5,48^{\mathrm{abB}}$ & $4,72^{\mathrm{abB}}$ & 5,09 \\
\hline Relação PI/PR & 1,59 & 1,56 & 1,43 & 1,56 & 1,94 & 1,61 \\
\hline$U G / R G$ relation & & & & & & \\
\hline Médias & 6,09 & 6,38 & 6,79 & 7,01 & 6,92 & 6,64 \\
\hline Means & & & & & & \\
\hline \multicolumn{7}{|c|}{ Consumo de $\mathrm{MO}(\mathrm{kg})$} \\
\hline & & & I intake ( & & & \\
\hline $\mathrm{PI}(U G)$ & $6,64^{\mathrm{cA}}$ & $6,89^{\mathrm{bcA}}$ & $7,11^{\mathrm{bcA}}$ & $7,60^{\mathrm{abA}}$ & $8,12^{\mathrm{aA}}$ & 7,27 \\
\hline $\operatorname{PR}(R G)$ & $4,16^{\mathrm{bB}}$ & $4,41^{\mathrm{abB}}$ & $4,99^{\mathrm{aB}}$ & $4,87^{\mathrm{abB}}$ & $4,19^{\mathrm{abB}}$ & 4,52 \\
\hline Relação PI/PR & 1,60 & 1,56 & 1,43 & 1,56 & 1,94 & 1,61 \\
\hline \multicolumn{7}{|l|}{$U G / R G$ relation } \\
\hline Médias & 5,40 & 5,65 & 6,05 & 6,24 & 6,15 & 5,90 \\
\hline Means & & & & & & \\
\hline
\end{tabular}

Médias seguidas pelas mesmas letras minúsculas (maiúsculas), na mesma linha (coluna), não diferem (P>0,05), pelo teste de Newman-Keuls

1 Período I = 18/11 a 19/12/96; II = 20/12/96 a 27/01/97; III = 28/01 a 04/03/97; IV = 05/03 a 08/04/97 e V = 09/04 a 13/05/97.

Means followed by same tiny (capital) letters do not differ $(P>0.05)$, by Newman-keuls test).

1 Period $I=$ from $11 / 18$ to $12 / 19 / 96 ; I I=$ from $12 / 20 / 96$ to $01 / 27 / 97 ; I I I=$ from $01 / 28$ to $03 / 04 / 97 ; I V=$ from $03 / 05$ to $04 / 08 / 97$ and $V=$ from $04 / 09$ to $05 / 13 / 97$.

R. Bras. Zootec., v.31, n.5, p.2123-2128, 2002 
Tabela 2 - Consumo diário de matéria seca e matéria orgânica por $100 \mathrm{~kg}$ de peso vivo dos animais dos tratamentos PI (pastejo irrestrito) e PR (pastejo restrito) e relação entre as médias dos dois tratamentos, nos cinco períodos de pastejo

Table 2 - Dry matter (DM) and organic matter (OM) daily intake per $100 \mathrm{~kg}$ live weight of the animals from the tratments UG (unrestricted grazing) and $R G$ (restricted grazing) and relation among the means for the two tratments, in five grazing periods

\begin{tabular}{|c|c|c|c|c|c|c|}
\hline \multirow{2}{*}{$\begin{array}{l}\text { Tratamentos } \\
\text { Treatments }\end{array}$} & \multicolumn{5}{|c|}{ Períodos } & \multirow{2}{*}{$\begin{array}{c}\text { Médias } \\
\text { Means }\end{array}$} \\
\hline & $\mathrm{I}$ & II & III & $\mathrm{IV}$ & $\mathrm{V}$ & \\
\hline \multicolumn{7}{|c|}{ Consumo de MS/ 100 kg PV (kg) } \\
\hline $\mathrm{PI}(U G)$ & $2,17^{\mathrm{aA}}$ & $2,14^{\mathrm{aA}}$ & $2,12^{\mathrm{aA}}$ & $2,19^{\mathrm{aA}}$ & $2,2^{\mathrm{aA}}$ & 2,16 \\
\hline $\operatorname{PR}(R G)$ & $1,48^{\mathrm{abB}}$ & $1,50^{\mathrm{abB}}$ & $1,68^{\mathrm{aB}}$ & $1,62^{\mathrm{aB}}$ & $1,31^{\mathrm{bB}}$ & 1,52 \\
\hline Relação PI/PR & 1,47 & 1,42 & 1,26 & 1,35 & 1,68 & 1,42 \\
\hline \multicolumn{7}{|l|}{$U G / R G$ relation } \\
\hline Médias & 1,83 & 1,82 & 1,90 & 1,91 & 1,75 & 1,84 \\
\hline \multicolumn{7}{|l|}{ Means } \\
\hline \multicolumn{7}{|c|}{$\begin{array}{c}\text { Consumo de } \mathrm{MO} / 100 \mathrm{~kg} \mathrm{PV}(\mathrm{kg}) \\
\text { OM intake/ } 100 \mathrm{~kg} \mathrm{LW}(\mathrm{kg})\end{array}$} \\
\hline $\mathrm{PI}(U G)$ & $1,93^{\mathrm{aA}}$ & $1,90^{\mathrm{aA}}$ & $1,89^{\mathrm{aA}}$ & $1,95^{\mathrm{aA}}$ & $1,95^{\mathrm{aA}}$ & 1,92 \\
\hline $\operatorname{PR}(R G)$ & $1,31^{\mathrm{abB}}$ & $1,33^{\mathrm{abB}}$ & $1,49^{\mathrm{aB}}$ & $1,44^{\mathrm{aB}}$ & $1,16^{\mathrm{bB}}$ & 1,35 \\
\hline Relação PI/PR & 1,47 & 1,42 & 1,26 & 1,35 & 1,68 & 1,43 \\
\hline$U G / R G$ relation & & & & & & \\
\hline Médias & 1,62 & 1,62 & 1,69 & 1,69 & 1,56 & 1,63 \\
\hline Means & & & & & & \\
\hline
\end{tabular}

Médias seguidas pelas mesmas letras minúsculas (maiúsculas), na mesma linha (coluna), não diferem entre si $(P>0,05)$, pelo teste de Newman-Keuls.

1 Período I = 18/11 a 19/12/96; II = 20/12/96 a 27/01/97; III = 28/01 a 04/03/97; IV = 05/03 a 08/04/97 e V = 09/04 a $13 / 05 / 97$.

Means followed by same tiny (capital) letters do not differ ( $P>0.05)$, by Newman-keuls test.

${ }^{1}$ Period $I=$ from $11 / 18$ to $12 / 19 / 96 ; I I=$ from $12 / 20 / 96$ to $01 / 27 / 97 ; I I I=$ from 01/28 to 03/04/97; $I V=$ from 03/05 to 04/08/97 and $V=$ from 04/09 to 05/13/97.

e no quinto período o maior consumo, que foram os períodos em que ocorreram a menor e a maior oferta de MVS por animal. A elevação verificada no consumo com o aumento da oferta de forragem está de acordo com os resultados de Stockdale et al. (1981). O consumo médio total diário de matéria orgânica (MO) mostrou comportamento idêntico ao observado para consumo de MS, conforme indicam as relações PI/PR para as duas variáveis (Tabela 1).

O consumo diário de MS por $100 \mathrm{~kg}$ de PV, para os animais em pastejo irrestrito, mostrou pequena oscilação nos cinco períodos de pastejo, com média de $2,16 \mathrm{~kg}$. Esse valor está dentro do intervalo apontado por Deresz \& Mozzer (1994), que comentaram que o consumo de MS, expresso em porcentagem do peso vivo, de animais em pastejo em capim-elefante, mostra grande variação, com extremos de 1,65 a $2,86 \%$ do peso vivo, principalmente para animais em crescimento. A relação média entre o consumo por $100 \mathrm{~kg}$ de PV observado entre os tratamentos PI/PR foi de 1,42 , situando-se pouco abaixo do valor médio de 1,62 observado entre os dois tratamentos, para consumo total diário de MS. Os valores de consumo de MO para os dois tratamentos guardam entre si relação semelhante à verificada para consumo de MS, refletindo a pequena variação observada nos teores de MO do capim-elefante nos diferentes períodos (Tabela 2).

Os períodos de maiores ganhos de peso (quatro e cinco) coincidiram com aqueles de maior consumo estimado de energia metabolizável. O ganho de PCVZ médio diário para os animais de pastejo irrestrito, nos cinco períodos, foi de $516,5 \mathrm{~g}$, tendendo a acompanhar a flutuação dos valores da relação MVS ofertada/ consumida, com correlação de $0,89(\mathrm{P}<0,05)$ entre as duas variáveis. Ganho diário semelhante foi verificado por Lima et al. (1969), para capim-elefante (512 g), em novilhos sob pastejo contínuo. Levantamento de Aguiar (1997), no Brasil Central, indicou ganhos diários médios, variando de 300 a $500 \mathrm{~g}$, em diferentes forrageiras. $\mathrm{O}$ ganho de peso estimado por hectare, baseado no ganho diário médio dos animais de pastejo irrestrito e na taxa de lotação equivalente a esses animais, foi de 432,5 kg nos cinco períodos de 
Tabela 3 - Consumo de energia metabolizável (EM) por unidade de tamanho metabólico ( $\left.\mathrm{kcal} / \mathrm{kg}^{0,75}\right)$, ganho de peso diário médio dos animais por tratamentos, relação matéria verde seca (MVS) ofertada/consumida e ganho de peso estimado por ha, por período experimental

Table 3 - Metabolizable energy (ME) intake per unit metabolic size, average daily weight gain of the animals per treatment, relation between green dry matter (GDM) offered/intaked and estimated weight gain per ha, per experimental period

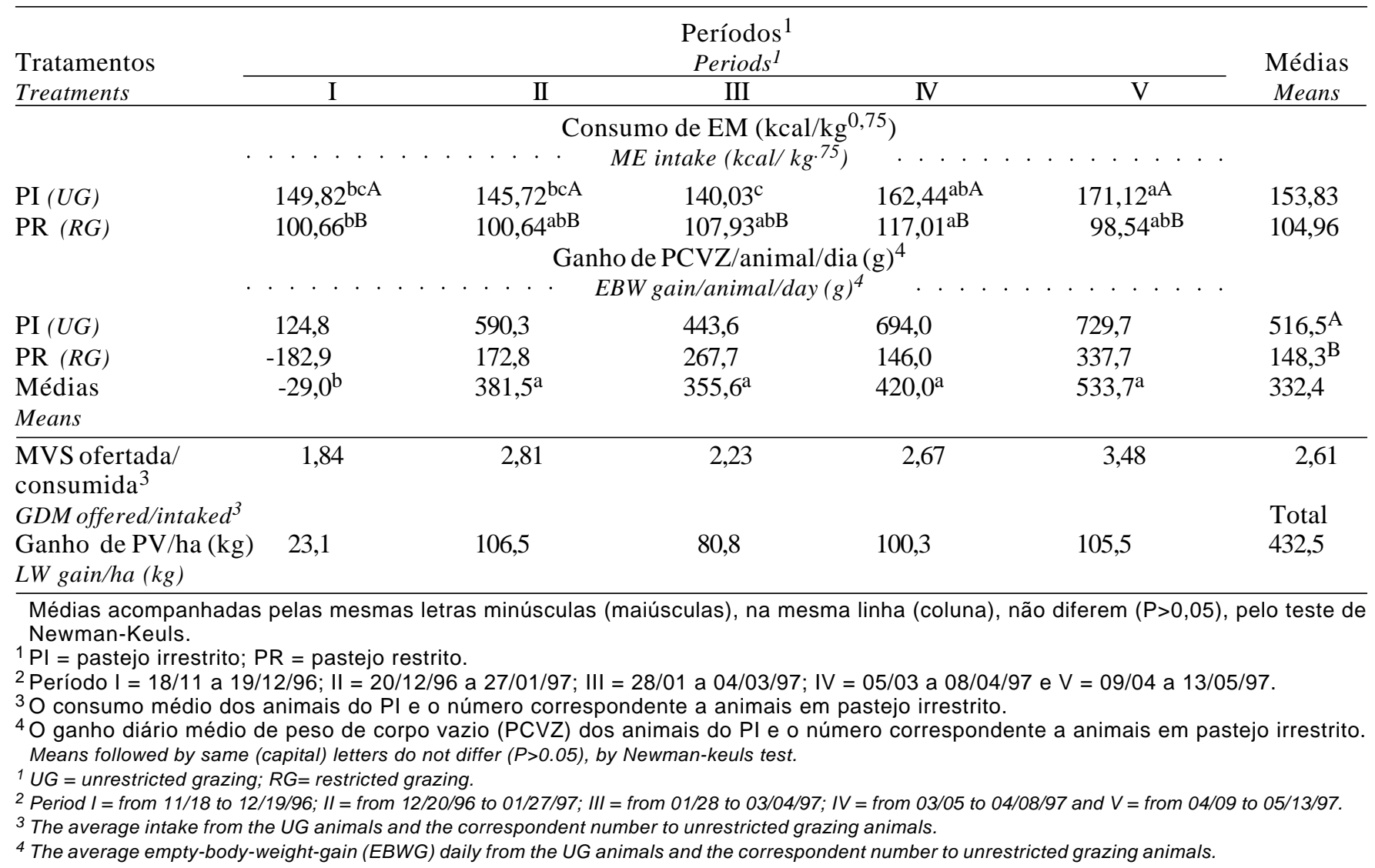

pastejo, situando-se dentro da faixa de ganhos verificada no Brasil para capim-elefante. Zimmer \& Euclides Filho (1997) citam ganhos anuais de 380; 580 e $626 \mathrm{~kg} / \mathrm{ha}$ em capim-elefante submetidos a três níveis de adubação. Os animais de pastejo restrito tiveram níveis baixos de ganho diário de peso de corpo vazio, com média de 148,3 g, mostrando que a restrição do tempo de pastejo permite manter o consumo alimentar próximo ao nível de mantença. Esse resultado indica que a restrição do tempo de pastejo a quatro horas diárias pode ser usada em pesquisas de determinação das exigências de energia de animais em regime de pastejo, nas quais um grupo de animais deve ingerir níveis de energia ligeiramente acima da mantença, enquanto outro deve ingerir energia ad libitum (Tabela 3 ).

\section{Conclusões}

Ocapim-elefante possibilitou ganhos satisfatórios de peso por animal e por hectare, em uma carga animal próxima a cinco novilhos, em fase de engorda, por hectare e pressões de pastejo de 4 a $7,8 \mathrm{~kg}$ de matéria verde seca por $100 \mathrm{~kg}$ de peso vivo.

Maiores ganhos de peso corresponderam aos períodos de maior consumo estimado de energia metabolizável por unidade de tamanho metabólico e mostraram associação positiva e estreita com os valores da relação MVS ofertada/consumida.

Quatro horas de pastejo diário garantiram consumo próximo à mantença, para uma pressão de pastejo de 4 a $7,8 \mathrm{~kg}$ de matéria verde seca por $100 \mathrm{~kg}$ de peso vivo. 


\section{Literatura Citada}

AGUIAR, A.P.A. Manejo da fertilidade do solo sob pastagemcalagem e adubação. In: CURSO DE MANEJO DE PASTAGEM, 2., 1997, Uberaba. Anais... Uberaba: PIAR, 1997. p.1-90.

BISHOP, J.P.; FROSETH, J.A. Improved techniques in esophageal fistulization of sheep. American Journal of Veterinary Research, v.31, n.8, p.1505-1507, 1970.

DERESZ, F.; MOZZER, O. L. Produção de leite em pastagem de capim-elefante. In: CARVALHO, M.M.; ALVIM, M.J.; XAVIER, D.F.; CARVALHO, L.A. (Eds.) Capim-elefante: produção e utilização. Coronel Pacheco, MG: EMBRAPACNPGL, 1994. p.195-215.

HARVEY, W.R. Mixed model least squares and maximum likelihood computer program (LSMLWM) versão PC-1, 1990.

HILLESHEIM, A. Fatores que afetam o consumo e perdas de capim-elefante (Pennisetum purpureum, Schum.) sob pastejo. Piracicaba: Escola Superior de Agricultura Luiz de Queiroz, 1987. 71p. Dissertação (Mestrado em Produção Animal) - Escola Superior de Agricultura Luiz de Queiroz, 1987.

LIMA, M.A.; MARTINELLI, D.; SARTINI, H. J. et al. Utilização de quatro gramíneas tropicais na produção de carne, em um solo típico latossolo roxo. Boletim da Indústria Animal, v.26 (único), p.199-214, 1969.

MINSON, D.J. Forage in ruminant nutrition. San Diego: Academic Press, 1990. 483p.

NATIONAL RESEARCH COUNCIL - NRC. Nutrient requirements of beef cattle. 6.ed. Washington, D.C.: 1996. $157 \mathrm{p}$.

NICOL, A.M.; KITESSA, S.M. Compensatory growth in cattle - revisited. Proceeding of the New Zeland Society of Animal Production, v.55, p.157-160, 1995.
SANTOS, F.A.P. Manejo de pastagem de capim-elefante. In: PEIXOTO, A.M.; MOURA, J.C.; FARIA, V.P. (Eds.) Volumosos para bovinos. 2.ed. Piracicaba: Fundação de Estudos Agrários Luiz de Queiroz, 1995. p.1-20.

STOCKDALE, C.R.; KING, K.R.; PATTERSON, I.F. et al. Hay supplements to overcome underfeeding of dairy cows. 1. Early lactation. Australian Journal of Experimental Agriculture, v.21, p.148-56, 1981.

STOCKDALE, C. R.; KING, K. R. Effect of stocking rate on the grazing behaviour and faecal output of lactating dairy cows. Grass and Forage Science, v.38, n.3, p.215-218, 1983.

TILLEY, J.M.A.; TERRY, R.A. A two-stage technique for the in vitro digestion of forage crops. Journal of the British Grassland Society, v.18, n.2, p.104-111, 1963.

VEIGA, J.B. Utilização do capim-elefante sob pastejo. In: CARVALHO, M.M.; ALVIM, M.J.; XAVIER, D.F.; CARVALHO, L.A. (Eds.) Capim-elefante: produção e utilização. Coronel Pacheco: EMBRAPA-CNPGL, 1994. p.165-193.

ZIMMER, A.H.; EUCLIDES FILHO, K. As pastagens e a pecuária de corte brasileira. In: SIMPÓSIO INTERNACIONAL SOBRE PRODUÇÃO ANIMAL EM PASTEJO, 1997, Viçosa, MG. Anais... Viçosa, MG: Universidade Federal de Viçosa, 1997. p.349-379.

Recebido em: 20/06/01 Aceito em: 07/05/02 